\title{
Clavien-Dindo classification and risk factors of complications after pancreaticoduodenectomy
}

\author{
Xiaoming Zou, Zhelin Yun, Shengbin Zhang, Jin Zhao, Bing Li* \\ Department of General Surgery, The Third Affiliated Hospital of Inner Mongolia Medical University, Baotou, China
}

Received: May 27, 2018

Accepted: July 15, 2018

Online Published: September 10, 2018

DOI: $10.14725 /$ dcc.v5n3p13

URL: http://dx.doi.org/10.14725/dcc.v5n3p13

\begin{abstract}
Objective: To analyze Clavien-Dindo classification and risk factors of complications after pancreaticoduodenectomy and investigate the relationship between the major risk factors and Clavien-Dindo classification of complications.

Methods: The retrospective case-control study was adopted. The clinical data of 200 patients who underwent pancreaticoduodenectomy at the Third Affiliated Hospital of Inner Mongolia Medical University from January 2010 to June 2015 were collected. The patients underwent Whipple procedure or pylorus-preserving pancreaticoduodenectomy according to the tumor site. Observation indicators included: (1) postoperative complications using Clavien-Dindo classification; (2) univariate and multivariate analyses: patients' basic information, surgery-related factors, pancreas-related factors; (3) relationship between independent risk factors and Clavien-Dindo classification of complications after pancreaticoduodenectomy. The chi-square test was applied to univariate analysis and categorical data. The comparison between groups was done by using independent samples nonparametric test (Kolmogorov-Smirnov Z), and multivariate analysis was done by using Logistic regression model.

Results: (1) Postoperative complications: Of 200 patients, 122 underwent Whipple procedure and 78 underwent pyloruspreserving pancreaticoduodenectomy, including 6 cases combined with vascular reconstructions and 1 case with RFA of liver tumors. Ninety-eight patients had postoperative complications, including 41 patients with no less than 2 types of complications. After surgery, pancreatic fistula was detected in 80 patients, including 42 cases with grade A, 28 cases with grade B and 10 cases with grade C; incisional infection in 29 patients; gastric retention in 24 patients; intra-abdominal infection in 16 patients; intraabdominal hemorrhage in 10 patients, including 8 patients receiving interventional treatment; biliary leakage in 7 patients and unplanned reoperation in 2 patients. Three patients were dead during hospitalization. The incidences of complications in grade I, II, III (III a and III b), IV and V of Clavien-Dindo classification were 28.00\% (56/200), 13.00\% (26/200), 5.00\% (10/200), $1.50 \%(3 / 200)$ and $1.50 \%$ (3/200). (2) Univariate and multivariate analyses: The results of univariate analysis showed that body mass index (BMI) and pancreas texture were risk factors affecting complications after pancreaticoduodenectomy $\left(\chi^{2}=6.483\right.$, $Z=-3.189, p<.05)$. The results of multivariate analysis showed that BMI $>23.9 \mathrm{~kg} / \mathrm{m}^{2}$ and soft pancreas were independent risk factors affecting complications after pancreaticoduodenectomy $(\mathrm{OR}=2.044,1.649,95 \%$ confidence interval: 1.212-3.447, 1.194-2.275). (3) The relationship between independent risk factors and Clavien-Dindo classification of complications after pancreaticoduodenectomy was analyzed. There were statistically significant differences between BMI or pancreas texture and Clavien-Dindo classification of complications after pancreaticoduodenectomy $\left(\chi^{2}=13.897,27.077, p<.05\right)$.

Conclusions: Clavien-Dindo classification of complications after pancreaticoduodenectomy contributes to comprehensive comparison and evaluation, and this type of classification in this study mainly refers to grade I and II. Reducing BMI and good management of pancreatic stump may improve Clavien-Dindo classification of complications after pancreaticoduodenectomy.
\end{abstract}

Key Words: Pancreatic diseases, Pancreaticoduodenectomy, Surgical procedures, Post-operative complications, Risk factors, Clavien-Dindo classification

\footnotetext{
${ }^{*}$ Correspondence: Bing Li; E-mail: libingice1980@163.com; Address: Department of General Surgery, The Third Affiliated Hospital of Inner Mongolia Medical University, Baotou, China.
} 
The ampullary tumor is one of common malignant tumors in China. Pancreaticoduodenectomy is still a standard surgical procedure for the treatment of ampullary tumors, but the prevention and treatment of post-operative complications and perioperative death has always been the focus that attracts current pancreatic surgeons. Although there are many studies on complications after pancreaticoduodenectomy and related risk factors, no uniform criteria were applied to the diagnosis of complications in most studies, with no classification to the severity of complications. Therefore, it is unable to make a comprehensive comparison and evaluation. This study retrospectively analyzed the clinical data of 200 patients undergoing pancreaticoduodenectomy from January 2010 to June 2015, and analyzed Clavien-Dindo classification of post-operative complications to explore risk factors affecting postoperative complications, aiming at reducing the incidence of post-operative complications.

\section{Data and methods}

\subsection{General information}

The retrospective case-control study was applied to this research. Clinical data of 200 patients undergoing pancreaticoduodenectomy were collected. There were 124 males and 76 females, aged 11 to 82 years, with an average age of 57 years. 72 patients had preoperative concomitant diseases, including 33 cases of hypertension, 32 cases of diabetes and 7 cases of coronary atherosclerotic heart disease. 20 patients had a past medical history of abdominal operations. 131 cases of patients had preoperative biliary tract obstruction, and 25 cases of patients received the treatment for the relief of jaundice. Information on tumor site was listed as follows: 79 cases of pancreatic head, 50 cases of end of biliary duct, 44 cases of duodenum, 26 cases of ampulla, and 1 case of pancreatic duct. This study was approved by Ethics Committee in our hospital. All patients and their families signed informed consent forms before surgery.

\subsection{Inclusion criteria and exclusion criteria}

Inclusion criteria were as follows: (1) Preoperative imageological examination indicated tumors on the duodenum, the pancreatic head or the lower part of biliary duct. (2) Patients had good cardiopulmonary function etc. to tolerate surgery. (3) There were no multiple metastases.

Exclusion criteria were as follows: (1) Patients with no less than two metastases; (2) Patients who received palliative operations.

\subsection{Surgical methods}

The patients underwent Whipple procedure or pyloruspreserving pancreaticoduodenectomy according to the tu- mor site. The pancreaticojejunostomy was performed according to the texture of pancreatic stump, the diameter of pancreatic duct and surgeons' experience. The drainage tube was routinely placed before or after the anastomosis of the pancreaticoduodenal region or the anastomosis of the cholangiojejunostomic region.

\subsection{Indicator observation}

(1) Postoperative complications: Refer to Clavien-Dindo classification standards for the diagnosis and classification of complications. ${ }^{[1]}$ (2) Univariate and multivariate analysis indicators: patients' basic information (age, body mass index [BMI], concomitant diseases, preoperative TBil, preoperative treatment for the relief of jaundice, preoperative Alb level, history of abdominal surgery), surgery-related factors (surgical methods, duration of surgery, intraoperative blood loss, perioperative blood transfusion) and pancreasrelated factors (pancreatic texture, the diameter of pancreatic duct). (3) Relationship between independent risk factors and Clavien-Dindo classification of complications after pancreaticoduodenectomy.

\subsection{Statistical analysis}

SPSS13.0 statistical software was applied to the statistical analysis. The chi-square test was applied to univariate analysis and categorical data. The comparison between groups was done by using independent samples nonparametric test (Kolmogorov-Smirnov Z). Multivariate analysis was performed by using Logistic regression model to calculate odds ratio (OR) value and $95 \%$ confidence interval for each factor. The difference $(p<.05)$ was of statistical significance.

\section{Results}

\subsection{Post-operative complications}

Of 200 patients, 122 underwent Whipple procedure and 78 underwent pylorus-preserving pancreaticoduodenectomy, including 6 cases combined with vascular reconstructions and 1 case with RFA of liver tumors. Ninety-eight patients had postoperative complications, including 41 patients with no less than 2 types of complications. After surgery, pancreatic fistula was detected in 80 patients, including 42 cases with grade A, 28 cases with grade B and 10 cases with grade $C$; incisional infection in 29 patients; gastric retention in 24 patients; intra-abdominal infection in 16 patients; intra-abdominal hemorrhage in 10 patients, including 8 patients receiving interventional treatment; biliary leakage in 7 patients and unplanned reoperation in 2 patients. Three patients were dead during hospitalization. The incidences of complications in grade I, II, III (III a and III 
b), IV and V of Clavien-Dindo classification were $28.00 \%$ (56/200), 13.00\% (26/200), 5.00\% (10/200), 1.50\% (3/200) and $1.50 \%(3 / 200)$.

\subsection{Analysis of risk factors}

The results of univariate analysis showed that BMI and pancreas texture were risk factors affecting complications after pancreaticoduodenectomy $\left(\chi^{2}=6.483, Z=-3.189, p<.05\right)$. See Table 1 for details.

The results of multivariate analysis showed that BMI >
$23.9 \mathrm{~kg} / \mathrm{m}^{2}$ and soft pancreas were independent risk factors affecting complications after pancreaticoduodenectomy $(p<.05)$. See Table 2 for details.

\subsection{Relationship between independent risk factors and Clavien-Dindo classification of complica- tions after pancreaticoduodenectomy}

There were statistically significant differences between BMI or pancreas texture and Clavien-Dindo classification of complications after pancreaticoduodenectomy $(p<.05)$.

See Table 3 for details.

Table 1: Univariate analysis on risk factors affecting complications in 200 patients undergoing pancreaticoduodenectomy

\begin{tabular}{|c|c|c|c|c|c|}
\hline Clinicopathological Factors & $\begin{array}{l}\text { Value } \\
\text { Assignment }\end{array}$ & $\mathbf{n}$ & $\begin{array}{l}\text { Cases of } \\
\text { Complications }\end{array}$ & Statistical value & $p$ value \\
\hline \multicolumn{6}{|l|}{ Age (years old) } \\
\hline - $<60$ & 0 & 111 & 56 & \multirow{2}{*}{$\chi^{2}=0.210$} & \multirow{2}{*}{$>.05$} \\
\hline$\cdot \geq 60$ & 1 & 89 & 42 & & \\
\hline \multicolumn{6}{|l|}{ BMI $\left(\mathrm{kg} / \mathrm{m}^{2}\right)$} \\
\hline$\bullet \leq 23.9$ & 0 & 122 & 51 & \multirow{2}{*}{$\chi^{2}=6.483$} & \multirow{2}{*}{$<.05$} \\
\hline - $>23.9$ & 1 & 78 & 47 & & \\
\hline \multicolumn{6}{|l|}{ Concomitant diseases } \\
\hline - None & 1 & 128 & 66 & \multirow{4}{*}{$\chi^{2}=1.446$} & \multirow{4}{*}{$>.05$} \\
\hline - Hypertension & 2 & 33 & 14 & & \\
\hline - Diabetes & 3 & 32 & 14 & & \\
\hline - Coronary atherosclerotic heart disease & 4 & 7 & 4 & & \\
\hline \multicolumn{6}{|l|}{ Preoperative TBil $(\mu \mathrm{mol} / \mathrm{L})$} \\
\hline$\bullet<170$ & 0 & 155 & 77 & \multirow{2}{*}{$\chi^{2}=0.127$} & \multirow[b]{2}{*}{$>.05$} \\
\hline$\bullet \geq 170$ & 1 & 45 & 21 & & \\
\hline \multicolumn{6}{|l|}{ Preoperative treatment for the relief of jaundice } \\
\hline - None & 0 & 175 & 86 & \multirow{2}{*}{$\chi^{2}=0.011$} & \multirow{2}{*}{$>.05$} \\
\hline - Yes & 1 & 25 & 12 & & \\
\hline \multicolumn{6}{|l|}{ Preoperative Alb Level (g/L) } \\
\hline$\bullet<35$ & 0 & 42 & 19 & \multirow{2}{*}{$\chi^{2}=0.301$} & \multirow{2}{*}{$>.05$} \\
\hline - $\geq 35$ & 1 & 158 & 79 & & \\
\hline \multicolumn{6}{|l|}{ History of abdominal surgery } \\
\hline - None & 0 & 180 & 87 & \multirow{2}{*}{$\chi^{2}=0.320$} & \multirow{2}{*}{$>.05$} \\
\hline - Yes & 1 & 20 & 11 & & \\
\hline \multicolumn{6}{|l|}{ Surgical procedure } \\
\hline - Whipple procedure & 0 & 122 & 57 & \multirow{2}{*}{$\chi^{2}=0.650$} & \multirow{2}{*}{$>.05$} \\
\hline - Pylorus-preserving pancreaticoduodenectomy & 1 & 78 & 41 & & \\
\hline Duration of surgery (min) & & & & & \\
\hline - $<360$ & 0 & 99 & 48 & & \\
\hline - $\geq 360$ & 1 & 101 & 50 & $\chi^{2}=0.021$ & $>.05$ \\
\hline Intraoperative blood loss (ml) & & & & & \\
\hline - $<1,000$ & 0 & 191 & 91 & & \\
\hline - $\geq 1,000$ & 1 & 9 & 7 & $\chi^{2}=3.123$ & $>.05$ \\
\hline Perioperative blood transfusion & & & & & \\
\hline - None & 0 & 158 & 75 & $\gamma^{2}=0.706$ & \\
\hline - Yes & 1 & 42 & 23 & $\chi^{2}=0.706$ & $>.05$ \\
\hline Pancreatic texture & & & & & \\
\hline - Hard & 1 & 111 & 45 & & \\
\hline - Moderate & 2 & 20 & 7 & $Z=-3.189$ & $<.05$ \\
\hline - Soft & 3 & 69 & 46 & & \\
\hline Diameter of pancreatic duct (mm) & & & & & \\
\hline$\bullet \leq 3.0$ & 1 & 106 & 52 & & \\
\hline - $>3.0$ and $<4.5$ & 2 & 35 & 16 & $\chi^{2}=0.232$ & $>.05$ \\
\hline - $\geq 4.5$ & 3 & 59 & 30 & & \\
\hline
\end{tabular}


Table 2: Multivariate analysis on risk factors affecting complications in 200 patients undergoing pancreaticoduodenectomy

\begin{tabular}{lllllll}
\hline Clinicopathological Factors & $\boldsymbol{b}$ value & Standard Error & Wald value & OR value & 95\% Confidence Interval & $\boldsymbol{p}$ value \\
\hline BMI & 0.715 & 0.267 & 7.184 & 2.044 & $1.212-3.447$ & $<.05$ \\
Pancreatic texture & 0.500 & 0.164 & 9.248 & 1.649 & $1.194-2.275$ & $<.05$ \\
\hline
\end{tabular}

Table 3: Relationship between BMI, pancreatic texture and Clavien-Dindo classification of complications after pancreaticoduodenectomy

\begin{tabular}{|c|c|c|c|c|c|c|c|c|c|}
\hline Clinicopathological Factors & $\mathbf{n}$ & Grade I & Grade II & Grade III a & Grade III b & Grade IV & Grade V & $\chi^{2}$ value & $p$ value \\
\hline \multicolumn{10}{|l|}{ BMI $\left(\mathrm{kg} / \mathrm{m}^{2}\right)$} \\
\hline - $\leq 23.9$ & 51 & 28 & 16 & 5 & 0 & 2 & 0 & 13.897 & $<.05$ \\
\hline - $>23.9$ & 47 & 28 & 10 & 3 & 2 & 1 & 3 & & \\
\hline \multicolumn{10}{|l|}{ Pancreatic texture } \\
\hline - Hard & 45 & 27 & 13 & 1 & 1 & 2 & 1 & & \\
\hline - Moderate & 7 & 2 & 5 & 0 & 0 & 0 & 0 & 27.077 & $<.05$ \\
\hline - Soft & 46 & 27 & 8 & 7 & 1 & 1 & 2 & & \\
\hline
\end{tabular}

\section{Discussion}

The incidence and severity of post-operative complications are commonly used critical indicators to evaluate the quality and safety of surgical procedures. Literature reports have shown that Clavien-Dindo classification standards are frequently used after radical operation for carcinoma of stomach. ${ }^{[2,3]}$ However, no uniform criteria were applied to the diagnosis of complications after pancreaticoduodenectomy in most studies, with no standardized classification to the severity of complications. Therefore, it is unable to make a comprehensive comparison and evaluation. In 2004, Dindo et al. ${ }^{[1]}$ proposed Clavien-Dindo classification standards on the basis of summarizing the clinical data of 6,336 cases of patients who underwent general surgeries. At present, this type of classification standards has been widely used to evaluate the severity of complications after abdominal operations etc. It was found that there were few literatures on Clavien-Dindo classification and risk factors of complications after pancreaticoduodenectomy. After summarizing the perioperative data of 700 patients and performing Clavien-Dindo classification of relevant complications, Braga et al. ${ }^{[4]}$ concluded that this type of new classification standards can not only accurately predict patients' postoperative condition, but also early screen out high-risk patients, helping surgeons develop individualized treatment options for the intra-operative and post-operative management of high-risk patients.

The results of this study showed that the incidence of complications with Clavien-Dindo classification (grade I to V) after pancreaticoduodenectomy was $49.00 \%$ (98/200), and the incidence of no less than 2 complications was $20.50 \%$ (41/200). This may be related to different surgical methods and patient choices. The results of this study indicated:
Clavien-Dindo grade I complications did not need to be treated, but grade II to IV complications should be treated with surgical interventions. In this study, 3 patients with grade $\mathrm{V}$ complications died of respiratory and circulatory failure.

The results of this study showed that patients with increased BMI had a 1.5-fold increased risk of post-operative complications in comparison to the control group. BMI was identified to be the most important risk factor for complications after pancreaticoduodenectomy. El Nakeeb et al. ${ }^{[5]}$ made a case-control study of 471 patients undergoing pancreaticoduodenectomy, and the results showed that a higher BMI could lead to a higher risk of post-operative complications. BMI is closely related to the fat content of the human body, and is also a major indicator of metabolic syndrome, which seriously affects the health of the body. It is a combination of factors that are interrelated in metabolism, associated with the occurrence and development of various chronic diseases such as cardiovascular disease, hypertension, diabetes, chronic obstructive pulmonary disease. ${ }^{[6]}$ A higher BMI can not only increase the risk of various metabolic dysfunctions associated with cardiovascular disease, but also increase the morbidity and mortality in some cancer patients. ${ }^{[7]}$ In addition, obese patients have an excessively long operation time and a massive intra-operative blood loss, which increase the incidence and mortality of short-term complications in patients after surgery. Whereas, short-term postoperative complications include incision fat liquefaction, incisional infection and anastomotic bleeding. It has been reported in literatures that the incidence of post-operative peritoneal abscess in obese patients with abdominal operations is 2 to 5 times than that in ordinary patients. The reason may be related to the anastomotic leak caused by high intraabdominal pressure in obese patients after surgery. ${ }^{[8]}$ 
It has been generally recognized that a soft pancreas and the diameter of pancreatic duct $\leq 3 \mathrm{~mm}$ are independent risk factors for pancreatic fistula after pancreaticoduodenectomy. ${ }^{[9-16]}$ Ramacciato et al. ${ }^{[17]}$ found that an increased BMI and the difference in pancreatic texture were also risk factors for post-operative pancreatic fistula. Ren et al. ${ }^{[18]}$ performed a retrospective analysis of 160 patients undergoing pancreaticoduodenectomy, and found that the diameter of pancreatic duct, duration of surgery and preoperative jaundice were independent risk factors for pancreatic fistula. The results of this study showed that there was no significant correlation between the diameter of pancreatic duct and the occurrence of post-operative complications, and the difference in pancreatic texture was an independent risk factor for complications after pancreaticoduodenectomy. Therefore, the pancreatic stump should be treated well as much as possible during surgery to reduce the occurrence of pancreatic fistula. The author considers that the relatively soft pancreatic stump can be made hardened by way of U-type suture

\section{References}

[1] Dindo D, Demartines N, Clavien PA. Classification of surgical complications: a new proposal with evaluation in a cohort of 6336 patients and results of a survey. Ann Surg. 2004; 240(2): 205 213. PMid: 15273542. https://doi.org/10.1097/01.sla. $0000133083.54934 . \mathrm{ae}$

[2] Lee JH, Park DJ, Kim HH, et al. Comparison of complications after laparoscopy-assisted distal gastrectomy and open distal gastrectomy for gastric cancer using the Clavien-Dindo classification. Surg Endosc. 2012; 26(5): 1287-1295. PMid: 22044981. https: //doi.org/10.1007/s00464-011-2027-0

[3] $\mathrm{Xu} \mathrm{WG}, \mathrm{Xu} \mathrm{H}, \mathrm{Ma} J$ J, et al. Analysis of risk factors and ClavienDindo classification of complications after laparoscopy-assisted gastrectomy. Chinese Journal of Digestive Surgery. 2016; 15(3): 228233.

[4] Braga M, Capretti G, Pecorelli N, et al. A prognostic score to predict major complications after pancreaticoduodenectomy. Ann Surg. 2011; 254(5): 702-708. PMid: 22042466. https ://doi .org/10. $1097 /$ SLA . Ob013e31823598fb

[5] El Nakeeb A, Hamed H, Shehta A, et al. Impact of obesity on surgical outcomes post-pancreaticoduodenectomy: a case-control study. Int J Surg. 2014; 12(5): 488-493. PMid: 24486933. https : //doi.org/10.1016/j.ijsu.2014.01.017

[6] Shao YK, Yao QY. Research progress in mechanisms by which bariatric surgery improves metabolism. Chinese Journal of Gastrointestinal Surgery. 2014; 17(7): 732-736. PMid: 25070457.

[7] Li Q, Zhang J, Zhou Y, et a1. Obesity and gastric cancer. Front Biosci (Landmark Ed). 2012; 17: 2383-2390. https://doi.org/ 10.2741/4059

[8] Bickenbach KA, Denton B, Gonen M, et al. Impact of obesity on perioperative complications and long-term survival of patients with gastric cancer. Ann Surg Oncol. 2013; 20(3): 780-787. PMid: 22976377. https://doi.org/10.1245/s10434-012-2653-3 and intermittent suture, which can reduce the mucosal tear in the surgical site to ensure the security of pancreatic and intestine anastomosis, thereby reducing the occurrence of post-operative pancreatic fistula.

In summary, Clavien-Dindo classification of post-operative complications is an important measure to comprehensively compare and evaluate the safety of pancreaticoduodenectomy. Complications after pancreaticoduodenectomy can be mainly divided into grade I and II according to this type of classification. Reducing BMI before surgery and good management of pancreatic stump during surgery may improve Clavien-Dindo classification of complications after pancreaticoduodenectomy.

\section{Conflicts of Interest Disclosure}

The authors have no conflicts of interest related to this article.
[9] Roberts KJ, Hodson J, Mehrzad H, et al. A preoperative predictive score of pancreatic fistula following pancreatoduodenectomy. HPB (Oxford). 2014; 16(7): 620-628. PMid: 24246089. https : //doi.org/10.1111/hpb.12186

[10] Chen ZM, Huang Q, Liu CH. Analysis on risk factors of pancreatic fistula after pancreaticoduodenectomy. Chongqing Medicine. 2015; 44(29): 4156-4158.

[11] Chen YY, Sun B, Jiang HC. Analysis of predisposing factors for pancreatic fistula after pancreaticoduodenectomy. Chinese Journal of Surgery. 2013; 51(8): 680-684.

[12] Liu W, Hua R, Sun YW, et al. Risk factors of pancreatic fistula after pancreaticoduodenectomy. Chinese Journal of Pancreatology. 2014; 14(5): 299-302.

[13] Ren YK, Han GS, Wang GC, et al. Clinical observation on the prevention of pancreatic fistula after distal pancreatectomy by embedding pancreatic stump. Chinese Journal of Pancreatology. 2013; 13(5): 333-334.

[14] Shi WG, Jiang ZW. Effectiveness analysis of Braun-anastomosis to prevent complications after duodenostomy. Chinese Journal of Endocrine Surgery. 2009; 3(3): 210-211.

[15] Zhao XH, Hong M, Lu XQ, et al. Application of pylorus preserving pancreaticoduodenectomy in operation for pancreatic cancer. Journal of Regional Anatomy and Operative Surgery. 2014; 23(4): 355357.

[16] Wang WK, Shen BY, Deng XX, et al. Risk factors of pancreatic fistula after pancreaticoduodenectomy. Chinese Journal of Digestive Surgery. 2014; 13(7): 531-534.

[17] Ramacciato G, Mercantini P, Petrucciani N, el al. Risk factors of pancreatic fistula after pancreaticoduodenectomy: a collective review. Am Surg. 2011; 77(3): 257-269. PMid: 21375833.

[18] Ren S, Liu P, Zhou N, et al. Complications after pancreaticoduodenectomy for pancreatic cancer: a retrospective study. Int Surg. 2011; 96(3): 220-227. PMid: 22216700. https://doi.org/10. $9738 / \operatorname{CC} 17.1$ 